\title{
Personalized Medicine: The Future of Health Care
}

\author{
Anna Meiliana ${ }^{1,2, *}$, Nurrani Mustika Dewi ${ }^{1,2}$, Andi Wijaya ${ }^{1,2,3}$ \\ ${ }^{1}$ Postgraduate Program in Clinical Pharmacy, Padjadjaran University, Jl. Eijkman No.38, Bandung, Indonesia \\ ${ }^{2}$ Prodia Clinical Laboratory, Jl. Cisangkuy No.2, Bandung, Indonesia \\ ${ }^{3}$ Postgraduate Program in Clinical Biochemistry, Hasanuddin University, Jl. Perintis Kemerdekaan Km.10, Makassar, Indonesia \\ *Corresponding author. E-mail: anna.meiliana@prodia.co.id
}

Received date: Oct 30, 2016; Revised date: Dec 5, 2016; Accepted date: Dec 15, 2016

\section{Abstract}

$\mathrm{B}$ ACKGROUND: Most medical treatments have been designed for the "average patients." As a result of this "one-size-fits-all-approach," treatments can be very successful for some patients but not for others. The issue is shifted by the new innovation approach in diseases treatment and prevention, precision medicine, which takes into account individual differences in people's genes, environments, and lifestyles. This review was aimed to describe a new approach of healthcare performance strategy based on individual genetic variants.

CONTENT: Researchers have discovered hundreds of genes that harbor variations contributing to human illness, identified genetic variability in patients' responses to different of treatments, and from there begun to target the genes as molecular causes of diseases. In addition, scientists are developing and using diagnostic tests based on genetics or other molecular mechanisms to better predict patients' responses to targeted therapy.

SUMMARY: Personalized medicine seeks to use advances in knowledge about genetic factors and biological mechanisms of disease coupled with unique considerations of an individual's patient care needs to make healthcare more safe and effective. As a result of these contributions to improvement in the quality of care, personalized medicine represents a key strategy of healthcare reform.

KEYWORDS: precision, medicine, genomic, proteomic, metabolomic

Indones Biomed J. 2016; 8(3): 127-46

\section{Introduction}

Since the genome project was conducted, we know that every individual has his/her own unique variations of the human genome, due to the combination of genetic variations and environment influence. Not all of those genome variations affect their state of health, but they could manifest in different individual responses to drugs treatment. Senior government officials, industry leadership, health care providers, followed by the public within the last decade, have testimony a steady embrace of genomic and personalized medicine. In genomic medicine, information from genomes and their derivatives (RNA, proteins, and metabolites) is used to guide medical decision making. Genomic medicine is a key component of personalized medicine, which is a rapidly advancing field of health care based on each person's unique clinical, genetic, genomic, and environmental information.(1)

Along the continuum from health to disease, genome information can provide DNA-based assessment for common complex disease, molecular indication for cancer diagnosis and prognosis, genome-guided therapy, dose selection, and much more for personal health care. This is moving fast in technological development, social and information revolution which will affect the health care way of thinking.(1) In simple word, genomic medicine is using information from genomes, either human or other organisms, and their derivatives to guide the medical decision making. Furthermore, it is now possible to examine a person's entire genome (or a fraction of it as you need) to assess 
individualized risk prediction and treatment decisions. Many patterns of gene expression across the entire genome are also now readily assayed. Thus, health and disease states can now be characterized by their molecular fingerprints to develop meaningful stratifies for patient populations and to elucidate mechanistic pathways based on genome-wide data.(1)

Personalized medicine is a broad and rapidly advancing field of health care that is informed by each person's unique clinical, genetic, genomic, and environmental information. Health care with personal medicine encircled could integrate and coordinate the evidence-based approach for patient care individually from health to disease. Personalized medicine needs multidisciplinary health care teams to reach its goal of promoting health and wellness, patients education and satisfaction, also disease prevention, diagnose and treatment. By genomic medicine, personalized medicine could be understanding molecular pathways of disease, therefore optimal health care strategies could be established in the earliest stage, and optimal medical care could be reached for better outcomes for each individual, to include treatments, medication types and dosages, and/or prevention strategies may differ from person to person, resulting in an unprecedented customization of patient care.(1) Personalized medicine, also referred to as individualized or precision medicine, is the practice of tailoring medical treatment to the individual characteristics of each patient.(2,3) Both physicians and patients highly expecting this enormous potential personalized medicine to give better clinical outcomes by moving away from a onesize-fits-all approach to a treatment strategy that are most likely to benefit each individual.(4)

\section{Building The Foundation for Genomic in Personalized Medicine}

On January 30, 2015, US President, Obama, announced funding for an Initiative in Precision Medicine (1) less than 3 years after a National Academy of Sciences committee report (2) made clear just how such an initiative could accelerate progress in medical care and research. By understanding precisely, what the distinguishing features of specific subgroups of patients are, we can better individualize therapies. This led to rapid improvement in technology that drives genetic discovery in human disease. We now can monitor our personal health and environment easier than ever, just using wearable activity trackers to metagenomic sequencing and direct-to-consumer genetic testing.
Human physiology is complex. There are some cases where the patient's symptoms cannot be ascertained, or the clinicians cannot gather enough data to decide, and these led to a guesswork inherent in the practice, that reduces the treatment strategies. The important contributor to this complexity is genetic. Though showing a similar set of symptoms, distinct genetic variants cause different respond to treatments. Without a mechanism to determine the underlying genetic cause of a set of symptoms, it might not be possible to determine which treatment will be most effective a priori.(7) Even when you know the cause of a condition, variants of unrelated genetic can affect treatment efficacy by altering the drugs pharmacokinetic. For example, patients with some genetic variants who are treated with traditional doses of azathioprine, an immunosuppressive drug for an extended period were known at risk of developing lifethreatening myelosuppression because the genetic variant prevent the drug from being properly metabolized.(8)

The goal of precision medicine is to enable clinicians to quickly, efficiently and accurately predict the most appropriate course of action for a patient. To achieve this, clinicians are given tools, in the form of tests and information-technology support, that are both compatible with their clinical workflow and economically feasible to deploy in the modern health-care environment. These tools help to simplify the process of managing the extreme biological complexity that underlies human disease. Then, a precision-medicine 'ecosystem' that link clinicians, laboratories, research enterprises and clinicalinformation-system developers together in new ways was developed to support the creation and clarification of these tools (Figure 1). These efforts will create a foundation of a continuously learning health-care system which was hope to accelerate the advance of precision-medicine techniques.(7)

Clinical laboratories with their information systems facilitate interpretations consolidation into reports and alerts, and the results applied with the help of Electronic Health Records (EHR) and associated systems, both when they are received and as the patient's condition and knowledge of the variants evolve. Patient-facing infrastructure or 'portals' provide individuals with access to their genetic data and, if appropriate, the ability to decide how they should be used, including whether to participate in research. At present, much of this infrastructure is at a very early stage of development. However, the infrastructural foundation for precision medicine is beginning to emerge.

Patients' role in supporting precision medicine also important. The precision medicine can be tailored to the 


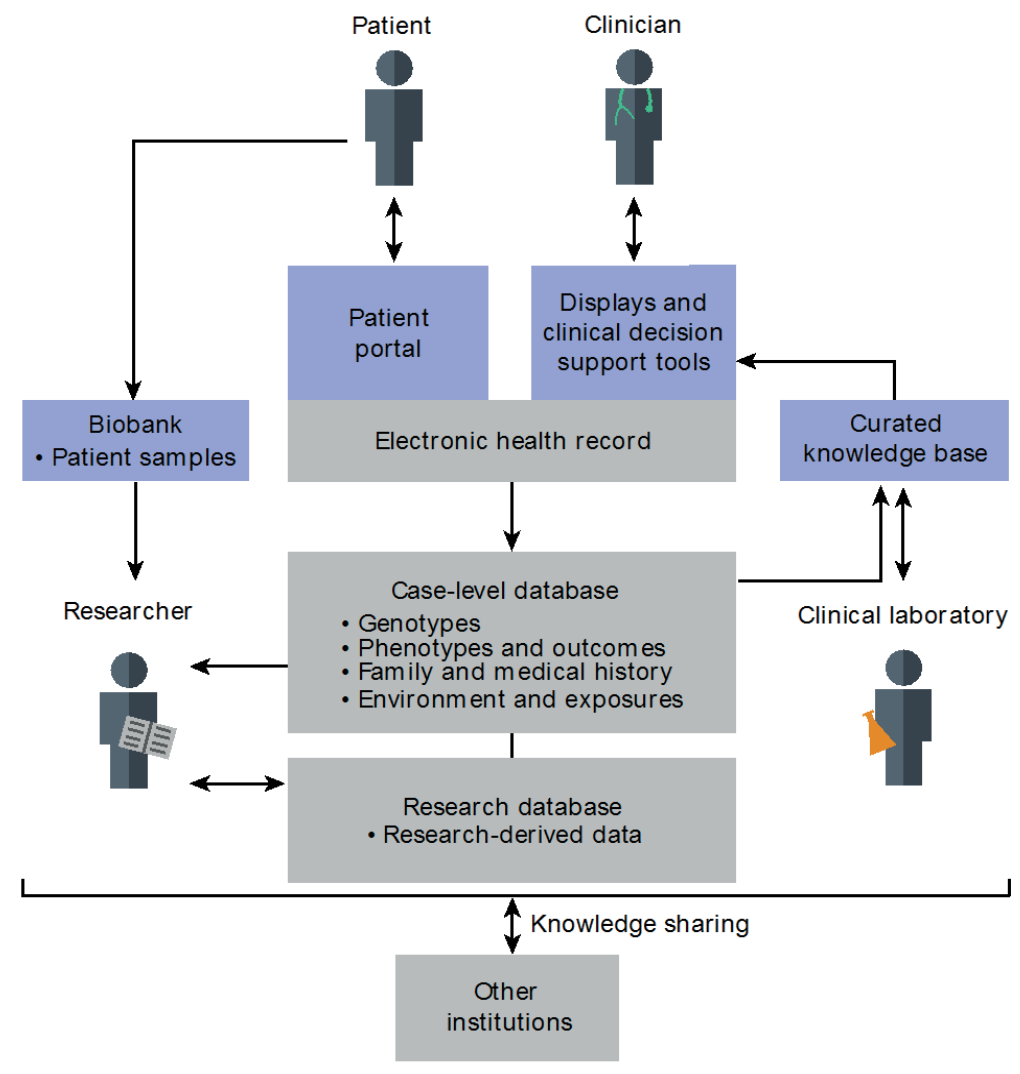

Figure 1. The precision-medicine ecosystem. The precision-medicine ecosystem contains building blocks that optimally connect patients, clinicians, researchers and clinical laboratories to one another.(7) (Adapted with permission from Nature Publishing Group). unique genetic make-up of each patient, by gathering as much information as possible from individual patient.(7) Clinicians usually take a role in patients' treatment order such tests for them.(8) Patients are obtaining an enlarge number of genetic results in the course of their care, and now they even can access direct-to-consumer testing, or through the help of someone who is not directly involved in their care.(7)

Clinicians gain access to patients' genetic information through tests. Tests have two components: a technical component that focuses on identifying which variants are present in the patient; and an interpretive component in which the implications of identified variants are assessed. In most scenarios, genetic testing is performed to determine either the cause of a specific indication or the most appropriate treatment.(9) Genome and exome sequences are possible to be obtained and stored, to be reused for multiple assessments perform over time even before disease manifests so that they can be interpreted and reinterpreted as indication arise.

EHRs are well positioned to be the apex of genetic information-technology support. They should serve as the clinician's gateway to all of the patient's information, including any genetic data. Information should be organized and displayed in a way that integrates with the clinician's workflow and facilitates diagnostic and treatment decisions. EHR and related systems can also provide clinicians with electronic clinical-decision support that provides extra information about a genetic test or result through an e-resource or InfoButton that links to electronic resources such as websites or databases. $(10,11)$ They can also issue pre-test and post-test pharmacogenomic warnings that highlight potentially adverse interactions between drugs and specific genetic variants. Pre-test will be suggested when a clinician is going to take an action that needs a genetic assessment information, but the patient's has no record of this assessment. Post-test alerts will be suggested when a clinician is going to take an action which is contraindicated with the patient's genetic profile.(7)

Clinical laboratories as the core of interpretative process provide either the evidence for individual variants and the case-level report with all potentially relevant variants in the context of the patient's presentation. New variants often found while performing genome sequencing, which must then be assessed. Many established variants also need to be assessed as a new knowledge rise. Laboratories and clinicians share variant- and gene-level assessment to increase the quality and efficiency of the variant assessment process.(12-16) The ClinGen program is building an authoritative central resource for use in precision medicine 
and research that defines the clinical relevance of genomic variants.

Several databases have been launched that share case-level data across broad disease areas. The National Center for Biotechnology Information (NCBI)'s database of Genotypes and Phenotypes places minimal restrictions on the types of case data that can be submitted and therefore serves as a generalized repository.(17) The International Cancer Genome Consortium (ICGC) and The Cancer Genome Atlas (TCGA) have each set up large repositories of somatic cancer sequencing data.(18,19) The American Society of Clinical Oncology (ASCO) is looking to incorporate the tracking of patient outcomes to enable a learning health care system in its CancerLinQ platform.(20)

\section{Systems Biology}

One of the great challenges for $21^{\text {st }}$ century medicine is to deliver effective therapies that are tailored to the exact biology or biological state of an individual to enable socalled 'personalized healthcare solutions.' Ideally, before the therapy started, this would involve a patient evaluating system that provides clinicians about the individual's correct drug and dose, or intervention. This evaluation concept approached on patient stratification, commonly according to some genetic features, be sub-classified to bio-features modeled in relation to the outcome. This stratification will be applied for personal therapy with a drug safety and efficacy model, as well as general healthcare involving optimized nutrition and lifestyle management.

Systems biology provides us with a common language for both describing and modeling the integrated action of regulatory networks at many levels of biological organization from the subcellular through cell, tissue and organ right up to the whole organism. The relatively new science of molecular epidemiology concerns the measurement of the fundamental biochemical factors that underlie population disease demography and understanding 'the health of nations' and this subject naturally lends it to systems biology approaches. Thus, personalized medicine and molecular epidemiological studies are certain to have a major role in future development of systems biology (Figure 2).(21)

Genetic variants predicted to severely disrupt proteincoding genes, collectively known as loss-of-function (LoF) variants, are of considerable scientific and clinical interest.(22) Proteins form the structural fabric of cells and underpin all metabolic processes and regulatory
INDIVIDUAL

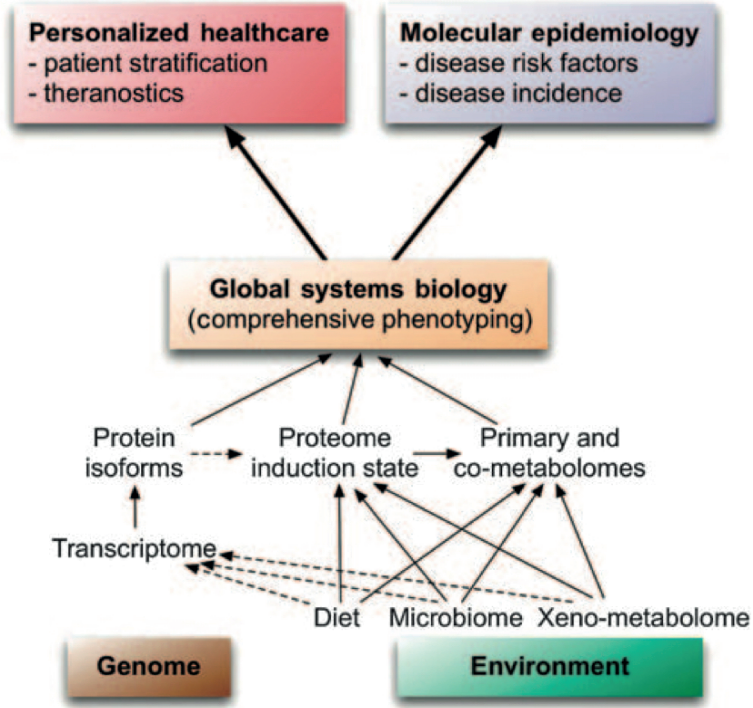

Figure 2. Relationships between systems biology, personalized healthcare and molecular epidemiology. Dotted lines indicate indirect connections or influences.(21) (Adapted with permission from Nature Publishing Group).

mechanisms. Protein properties, including abundance levels, protein-protein interactions, post-translational modifications subcellular localization patterns and protein synthesis and degradation rates, are all highly dynamic and can change rapidly during the course of biological processes, such as cell proliferation, cell migration, endocytosis and development. Therefore, understanding protein structurefunction relationships in cell biology not only requires the identification of proteins but also the detailed analysis of the protein properties that constitute the dimensions of the proteome.

Several studies in cellular processes have involved multi-dimensional analysis of protein properties to understanding cell and tissue biology better. Many of these have been aided by developments in mass spectrometry (MS)-based analysis, enabling higher sensitivity and a higher dynamic range of quantification.(23-26) In addition, over the past decade, biochemical and cell biological fractionation, such as chromatography or centrifugationbased separations, have increased in efficiency and resolution. Thus, multiple separations can now more easily be combined for sequential multidimensional proteome analysis (Figure 3).

MS-based proteomics was now developed to enable the multiple properties measurements of thousands proteins, including their abundance, isoform expression, turnover rate, subcellular localization, post-translational modifications and interactions and will be completing with new data analysis, 


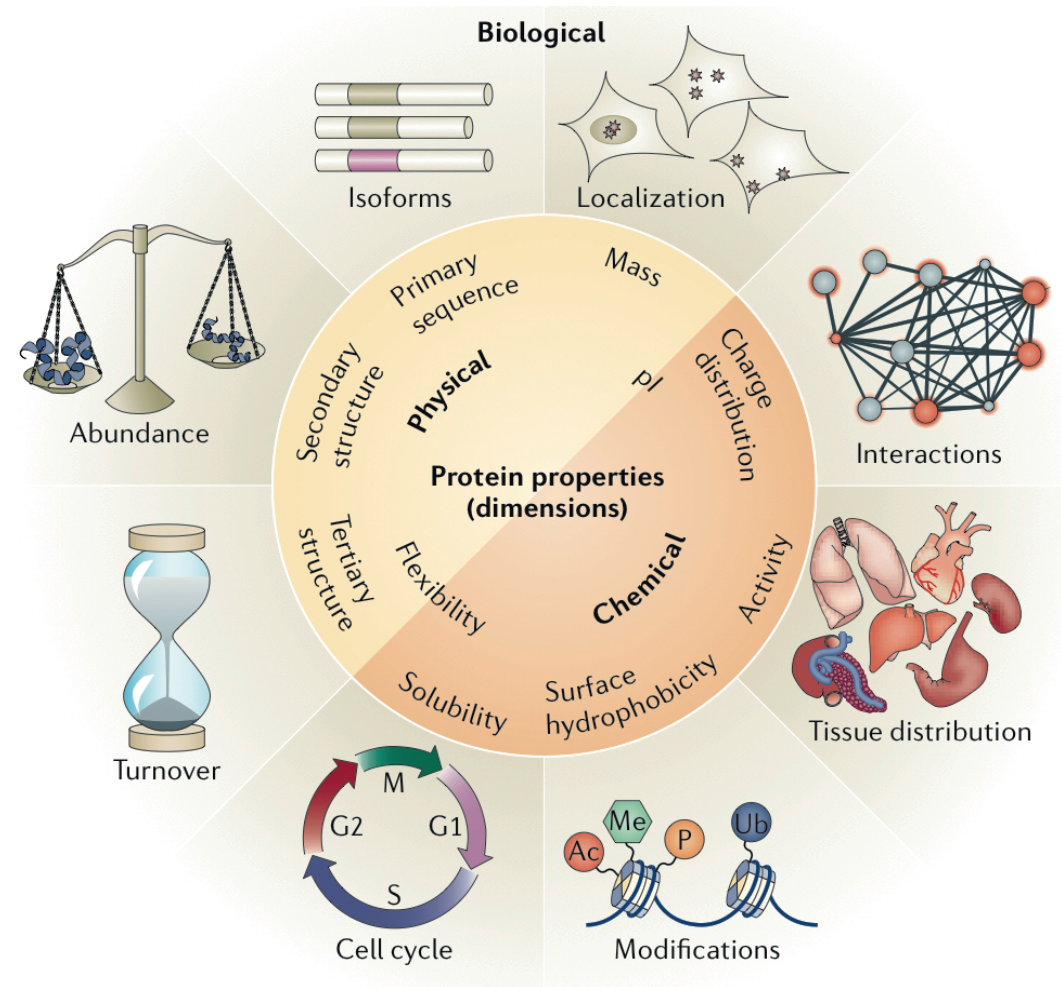

Figure 3. Multidimensional proteomic analysis of cells and tissues. Proteins can have many different properties (dimensions) that are either largely physically (yellow shaded area), chemically (orange shaded area) or biologically (beige shaded area) relevant.(27) (Adapted with permission from Nature Publishing Group). integration and visualization tools as well as data-sharing resources. Together, these advances in the multidimensional analysis of the proteome are transforming our understanding of various cellular and physiological processes.(27) This will not only be important to magnify our understanding of basic cellular physiology and regulation but also for future advances in medicine and drug development.

Personalized healthcare and molecular epidemiology are thus effectively two sides of the same 'systems biology coin'; the essential differences are with respect to the type of medical endpoints or outcomes that are to be modeled (Figure 2). Metabolomics offers a practical approach to measuring the metabolic end points that link directly to whole system activity and metabolic profiles are determined by both host genetic and environmental factors.(28) Metabolomics is an emerging field and is broadly defined as the comprehensive measurement of all metabolites and low-molecular-weight molecules in a biological specimen. Because metabolomics affords profiling of much larger numbers of metabolites than are presently covered in standard clinical laboratory techniques, and hence comprehensive coverage of biological processes and metabolic pathways, it holds promise to serve as an essential objective lens in the molecular microscope for precision medicine.(29) Practically, not alike genomic or proteomic methods, metabolomics presents a significant analytical challenge, due to its aim in measuring disparate physical molecule properties (e.g., ranging in polarity from very water soluble organic acids to very nonpolar lipids).(30) Accordingly, comprehensive metabolomic technology platforms typically take the strategy of dividing the metabolome into subsets of metabolites, often based on compound polarity, common functional groups, or structural similarity, and devise specific sample preparation and analytical procedures optimized for each, as illustrated in Figure 4. The metabolome is therefore measured as a patchwork of results from different analytical methods.

Metabolomics evolve rapidly nowadays, aim for an ideal comprehensive measurements of all endogenous metabolites in a cell or body fluid, and providing a functional readout of human body's physiological state. Hemostasis of key lipids, carbohydrates, or amino acids can change due to the genetic variants. Their involvement directly in metabolic conversion modification are not only expected to display much larger effect sizes, and also expected to provide access to the biochemical context of such variations, in particular when enzyme coding genes are concerned.(31) Now, metabolomics is on the level of technology refinement, and we are still determining what actually constitutes the human metabolome, while the expectation of small molecules finding in the human body exceeds 19,000.(32) This number includes not only metabolites directly linked to endogenous enzymatic activities encoded by the human genome, but also those derived from food, medications, the microbiota 


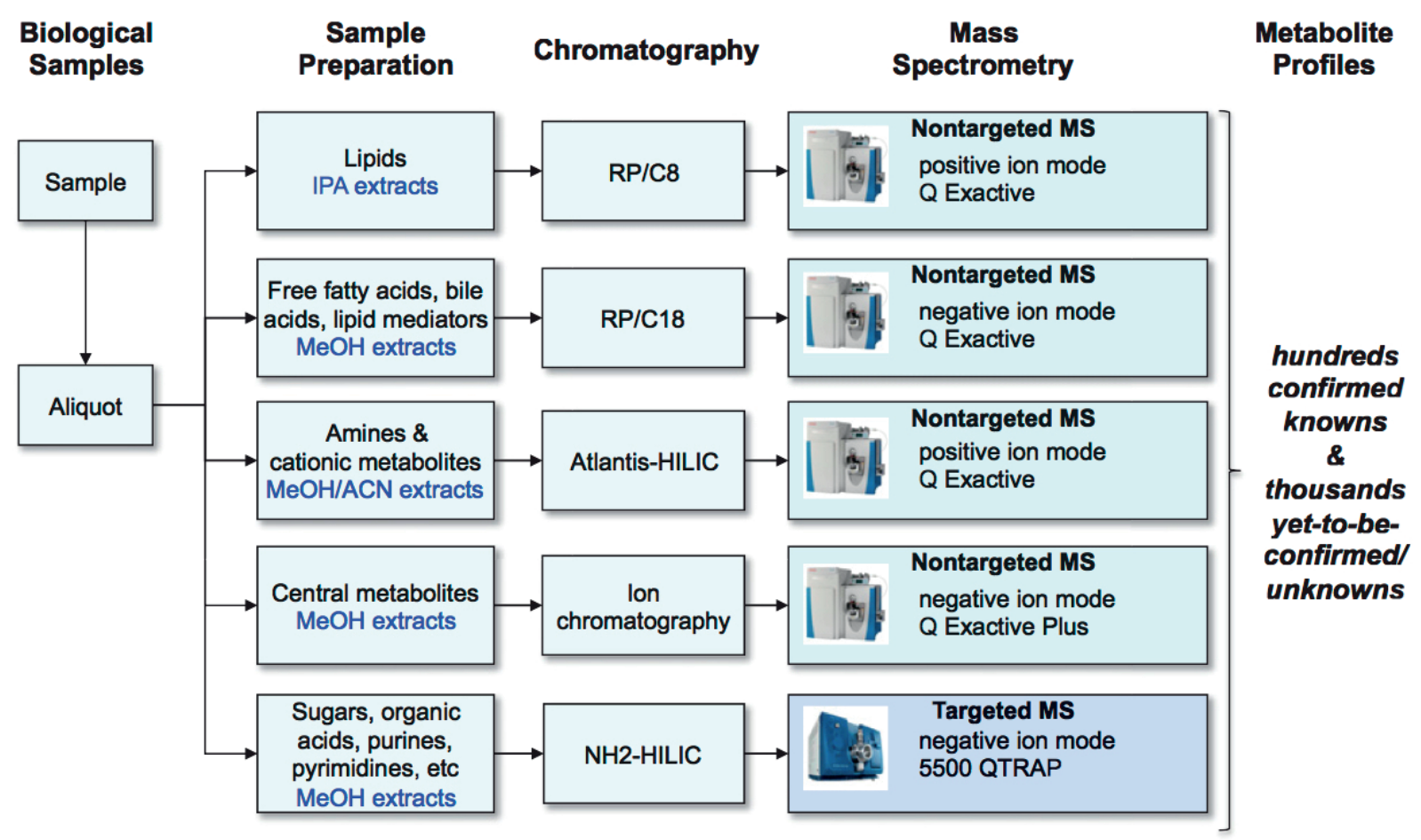

Figure 4. Illustration of the liquid chromatography-MS (LC-MS)-based metabolomics platform used at the Broad Institute of MIT and Harvard.(29) (Adapted with permission from Cold Spring Harbour Laboratory Press). IPA: isopropanol; MeOH: methanol; AcCN: acetonitrile; RP/C8: C8 reversed phase; RP/C18: C8 reversed phase; HILIC: hydrophilic interaction chromatography.

that inhabit the body, and the environment. Our dependence on diet as a source for nine of the 20 amino acids for which there are codons in the human genome but no endogenous biosynthetic route is an example that highlights why it is important to account for "exogenous" metabolites in our study of the metabolome.(29)

The discovery of specific markers for diseases and drug pharmacodynamics, as well as metabolite profiles, in relation to external environment and disease risk could enhance the potential of precision medicine. Current metabolomics technologies can enable more rapid discovery and validation of metabolic indicators of disease. Techniques used in metabolomics, such as liquid chromatographymass spectrometry (LC-MS), can routinely measure tens to hundreds of metabolites with excellent precision and are suitable for discovery studies in human cohorts. Confidence comes from experience with recent applications to find early metabolic indicators of disease in longitudinal cohorts years before symptoms are clinically apparent, for example, in pancreatic cancer (33), type 2 diabetes (34-36), memory impairment (37), and many other conditions. Many metabolomics studies provide novel view about relationship between diet and diseases, provoke applied work such as observing the association between elevated branched chain amino acids and obesity to insulin resistance.(38) System biology genomic to phenotype is shown by Figure 5 .

\section{Human Phenotyping}

A phenotypic abnormality is defined in medical settings as a deviation from normal morphology, physiology, or behavior, and good phenotyping is a cornerstone of a doctor's daily work.(39) Progress in information technologies together with next-generation sequencing (NGS), proteomics, and metabolomics are bringing about a paradigm shift in translation research and clinical care. Physicians and patients in the future will allow accessing a large-scale data to help them stratifying and improving the medical treatments. Provided correct and up-to-date information with sufficiently detailed and accurate phenotypic description will support the best treatment selection. $(40,41)$

The term "phenotype" used in medical context refers to some deviation from normal morphology, physiology, or behavior. This phenotype analysis plays a key role clinical and medical practice as well as research, but these descriptions in clinical notes or medical publications often were imprecise. Deep phenotyping can be defined 


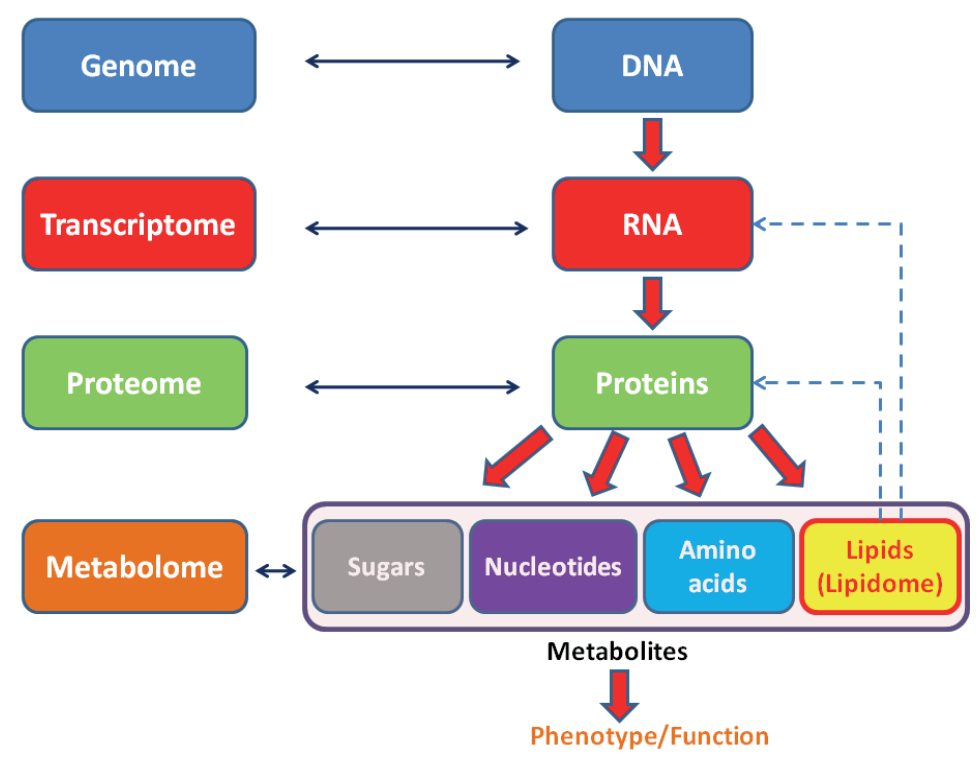

Figure 5. Genomic to phenotype (system biology).

as the precise and comprehensive analysis of phenotypic abnormalities where an individual assessment are performed for detail components of the phenotype observation and description.(42)

The International Standards for Cytogenomic Arrays Consortium has promoted standards for chromosomal microarray analysis and phenotypes and currently collected data on over 28,500 cytogenomic array investigations (43), and is thus one of the first examples of a Human Phenome Project covering a specific area of genetic medicine. The Personal Genome Project was aimed to involve 100,000 informed consent-signed general public participants to share their genome sequence and some personal and phenotypic information. Here, a prototype project involving metabolomic phenotyping coupled to the targeted analysis of a set of genes known to be involved in metabolic disturbances is presented.(44)

Deep phenotyping generally performed in such a way as to be computationally accessible. Using the common biological basis stratification, precision medicine intends to reconcile the best available care into the disease subclasses. These comprehensive discoveries and their translation into clinical care, critically need a computational resource to capture, store and exchange deep phenotypic data. A sophisticated algorithm will be required to integrate this deep phenotype data with genomic variation and additional clinical information.(42)

A "traditional" method of retrieving phenotype data from the medical literature or ERH for computational analysis is text mining. However, the overwhelming majority of clinical descriptions in the medical literature are simply natural language text, and thus automated searching, analysis, and integration of medical information from databases such as PubMed remains challenging.(45) To overcome those limitations, a structured, comprehensive, and well-defined phenotyping terminology is established. The Human Phenotype Ontology (HPO), available at www. human-phenotype-ontology.org, provides a set of more than 11,000 terms describing human phenotypic abnormalities. They describe the concepts of human phenotypes as well as a logical (computational) representation of the interrelationships between the terms.(41)

The rapid growth of sequencing technologies has greatly contributed to our understanding of human genetics. Yet, despite this growth, mainstream technologies have not been fully able to resolve the diploid nature of the human genome, including the method to determine allele-specific methylation patterns in a human genome and identify hundreds of differentially methylated regions that were previously unknown.(46) Besides differential methylation studies, haplotype information has applications in many areas of genomics, including (i) the analysis of disorders affected by compound heterozygosity, such as blistering skin (47), cerebral palsy (48), deafness (49) and others (50); (ii) population genetics, where population-specific haplotype blocks are currently resolved using loweraccuracy statistical methods (51); (iii) the detection of structural variations, which has been shown to benefit from phase information (52); (iv) the matching of hosts and donors in organ transplantation based on the human leukocyte antigen (HLA) region of the genome (53); (v) the evolution of genomes across species (54). 
To understand the relationship between genotype and phenotype, we need a haplotype-resolved information for the human genome, because we might find that a different configuration of exactly the same set of variants can sometimes result in different outcomes with regard to phenotype and disease susceptibility.(50) This information has typically been obtained by mapping sequence reads back to the human genome reference (55), and such methods cannot be applied to species for which a reference genome is not available. Advanced NGS technology and numerous bio-informatics techniques (56-58) have been developed and applied to the production and analysis of large-scale human sequence data in many individuals (59-62) and international projects (63-67). However, NGS technology give a shortread format of mixed DNA fragments derived from a pair of diploid chromosomes, and this posing challenges for determining haplotype information.(66)

Several computational and experimental methodologies have been developed to obtain haplotype information, including (i) population-based statistical phasing by integration of unrelated individual data $(63,67)$; (ii) trio-based phasing applying Mendel's law of inheritance (68); (iii) phasing by direct usage of sequence reads information (59); (iv) experimental phasing that includes the use of various forms of polymerase chain reaction (PCR), atomic force microscopy with carbon nanotubes (69) and hybridization of probes to single DNA molecules (70,71); (v) physical methods involving the initial preparation of haploid genomic material, for which the haplotype origin is distinguishable after sequencing $(52,53,72)$. Stratified medicine could be simply defined as tailoring of medical treatment to the individual characteristics of each patient. It doesn't mean the drugs or medical devices were created individually for each patient, but rather about classifying patients into a stratified subpopulation based on their uniqueness and their susceptibility (or severity of) a particular disease and their response to a specific treatment. Preventive or therapeutic interventions can then be concentrated on those who will benefit, sparing expense and side effects for those who will not". It also involves the development, validation and use of companion diagnostics to achieve the best outcomes in the management of a patient's disease or future prevention.(73)

Exploiting continuing scientific advances in genomics, molecular biology and medical technologies to detect and classify diseases more objectively lies at the heart of stratified medicine. Many reports apply the term "stratification" for describing this molecular sub-classification of disease and disease susceptibility based on both biomarkers and phenotypic descriptions. It was crucial to note that this stratification is not limited to molecular technologies. Actual and future advances in these areas are leading to an increase in the efficiency and precision of drug use, dose selection and diagnostic discovery and development.(73)

In earlier 2015, tech giant Apple announced the launch of its ResearchKit. The ResearchKit is a mobile platform that taps into the iPhone's 700 million global users to find individuals interested in participating in human research studies. The first five apps included in the kit enable users to enroll in observational studies on Parkinson's (mPower app), cardiovascular health (MyHeart Counts), breast cancer (Share The Journey), asthma (Asthma Mobile Health) and diabetes (GlucoSuccess). The studies are being run in conjunction with 17 different partner research institutions and foundations, many of which are US-based. ResearchKit is the latest of several ambitious initiatives that seek to harness the convergence of mobile technology, wearable sensors for measuring phenotypic markers, and highly sensitive technologies for measuring genomic, epigenetic, proteomic and metabolic markers in blood, stool and tissue. Ultimately, the harnessing of these technologies with computational platforms to store, share, filter and analyze the data will make it possible to collect markers of health and disease data for entire human populations, opening new possibilities for biomedical research.(74)

Another strength of platforms driven by mobile technology is that they offer the ability to monitor phenotypes in a longitudinal manner. In the case of the mPower app, trial participants could track how a disease affects gait, tremors, mood, cognition, fatigue, speech and sleep on a daily basis. This gives a chance about revealing new informative patterns of markers regarding the disease progression and severity. Sufficient sample sizes was expected to facilitate an adequate statistical power analysis that may enhance our ability to stratify diseases, which are currently defined on the basis of imprecise symptoms/phenotypes, into more finely described subgroups. Indeed, a major aim of the $100 \mathrm{~K}$ Wellness project (currently scaling up to 1,000 individuals) the Personal Genome Project, Human Longevity and the Google Baseline Study is to compare the transition of individuals from wellness to disease. This is significant because in many cases we are unclear as to what is meant by a 'healthy person.' Finer and more exact categorization of phenotypes will mean that we will have the potential to diagnose disease faster and earlier than before, which in itself is likely to make interventions more effective.(74) 


\section{Pharmacogenomics}

After decades of exploration, approximately 20 genes of inherited variations have been identified which affect about 80 medications and are actionable in the clinic. Some somatically acquired genetic variants direct the choice of 'targeted' anticancer drugs for individual patients. The focus of current efforts for appropriate are shifting from discovery to the implementation of an evidenced-based strategy to improve the use of medications, thereby providing a cornerstone for precision medicine.(75) Pharmacogenomics focuses on the identification of genetic variants that influence drug effects, typically through alterations in pharmacokinetic, that is how the drug is absorbed, distributed, metabolized or eliminated, or pharmacodynamics, by modifying its target or by perturbing the biological pathways that shape a patient's sensitivity to its pharmacological effects. Most genetic variations either inherited from parents or changed de novo identified in germline DNA, and alter the function of gene products. Differently, in cancer, patient's response to the treatment was affected by both inherited and somatically acquired variants. In infectious diseases, genetic variation can affect a pathogen's sensitivity to antimicrobial drugs. Genome interrogation technology for analytical approaches has come to advance, lead to the evolution of a discovery model from gene studies candidate to a new finding of agnostic genome-wide analyses in specific drug-response phenotypes patients population, for example, toxicity or desired pharmacological effects. In fact, the genome interrogation technologies currently are sufficiently robust that makes it harder to define the drug-response phenotype in pharmacogenomics research. Once a pharmacogenomic relationship has been discovered and validated, there are many obstacles to translating it into clinical practice. Such translation requires that effective, alternative therapy is available for those with 'high-risk' genotypes, as well as improvements to health care systems, structured approaches to guide prescribing (for example, algorithms), and implementation of point-of-care electronic clinical decision support, to make it feasible to utilize genetics appropriately to guide drug prescribing.(75)

More than 1,200 individual molecular entities have been approved as drugs by the US Food and Drug Administration (FDA) (76), the European Medicines Agency (EMA) (77) or by Japan's Pharmaceuticals and Medical Devices Agency (PMDA) (78). Although about $15 \%$ of the medications approved by the FDA and EMA contain pharmacogenomic information on their label, only a subset of the corresponding pharmacogenes is deemed actionable.(77,79) As summarized, medications have actionable germline pharmacogenetics. These correspond to Clinical Pharmacogenetics Implementation Consortium (CPIC) level A or B gene-drug pairs for which genetic information should or could be used to change the prescription pattern of the relevant drug.(80) In the United States, these medications constitute $18 \%$ of all prescriptions, which indicates that pharmacogenomically high-risk medications are slightly overrepresented in highly prescribed medications.(81) So far, only 16 of the roughly 19,000 human genes are considered to be clinically actionable for germline pharmacogenomics.(80) Prescription medication are unlikely to be useful in most human germline genetic variation, as well as pharmacogenomics can not be useful enough for improving the prescription of the majority of drugs. However, for the relatively small set of medications where genomics can be actionable, more widely genetic testing and appropriately deploying of it in the clinic could optimize the prescribing.(75)

Some barriers still be the obstacle in wide spreading the use of pre-emptive multigene panels to guide the drugs prescription, such as the lack of incentives for clinicians to conduct tests or implement procedures that might prevent adverse events. There are relatively few studies that prove the cost-effectiveness of pharmacogenetic testing.(82) Although a multigene panel approach is less expensive than ordering tests for one pharmacogene at a time, there are no data to assess the cost-effectiveness of the panel approach when implemented early on in life and used throughout a patient's lifetime. Another barrier is the fact that financial reimbursement for preventive-medicine services or for preemptive screening usually was not provided by most healthcare system. $(83,84)$

As deep sequencing becomes more widespread, further variants will be discovered in pharmacogenes.(85) The challenge will be to catalog and annotate these variants. Given the importance of rare variants for both inherited (86) and cancer-related pharmacogenes, publicly available and easily updatable resources such as PharmGKB, ClinGen and ClinVar will be essential for providing the computational clinical-decision support in health care record systems with up-to-date recommendations that are based on genetic-test results.(87-89) Clinicians are accustomed to making prescribing decisions on the basis of patient characteristics such as age, kidney or liver function, drug-drug interactions and personal preferences. This data should be compiled with optimal clinical-decision support to create a well-organized compilation of one's characteristics 
matched with evidenced-based choices on medications and doses. Pharmacogenomics was hope to be a component of evidence-based precision medicine, with the improved of clinical-decision support and pharmacogenomic testing continues to grow accelerate with clinical implementation of pharmacogenomics.

\section{Personalized Medicine Toolbox}

Next generation technologies (NGT) proposed huge improvements not only in cost efficiency, accuracy, robustness, but also in the amount of information they provide. Unique combination of microarrays together with high-throughput sequencing platforms, digital droplet PCR, and other technologies will offer a more desirable performance. As stronger evidence of genetic testing's clinical utility influences patterns of patient care, demand for NGT testing is increasing. This will challenge the clinical laboratories to provide NGT-based protocols aligned with the traditional tests, while the urgency, clinical importance, and breadth of application in molecular oncology, as well as more integration of genetic tests into synoptic reporting keep increasing.(90)

The advances in NGT technologies coalesced with the accelerated discovery of the genetic basis of human diseases in parallel feeding the molecular genetic testing to be rapidly expanding, and makes possible to convert cumbersome Sanger-based assays to be a streamlined and less costly, comprehensive targeted gene panels with the application of whole exome sequencing (WES) and whole genome sequencing (WGS) so that molecular diagnosticians could easily examine the known genes responsible for target phenotype(s) and to identify previously unrecognized causes for the heritable disorder for which the test was indicated. Such testing also identifies incidental findings, or off-target sequence alterations unrelated to the reason for testing, that could affect the participant's health now or in the future. Readily interpretable test reports, however, still not easy to produce due to exome and genome sequencing which increasing the test complexity. In addition, new "meaningful use" components of Medicare and Medicaid EHR Incentive Programs (from Centers for Medicare and Medicaid Services, in 2013) permit patients to directly access results from all clinical laboratory tests, which create a new audience that may struggle to interpret complex genomic reports. With any rapidly evolving technology comes growing pains and caveats. Clinical laboratories that report results from exome or genome sequence data must be able to communicate the outcomes of those efforts effectively.(91)

The first human genome costs $\$ 3$ billion and took 13 years to sequence; today such an undertaking costs closer to $\$ 1,000$ and takes only days, making large-scale genetic analysis feasible and affordable. Short- and longread sequencers all the time is known as an established workhorses in biomedical research, and now their uses are expanding into clinical applications and beyond. Most notably, the combination of high-throughput genotyping with measurements of other markers of health and disease is opening up the area of precision medicine.(92)

Although NGS (Table 1) platforms have become an established tool in the research arena, a highly anticipated area of growth in the research market is the large-scales genotyping of populations. In 2012, UK Prime Minister David Cameron announced a project to sequence the genome up to 100,000 people and use their genomic information in treatment and studies of cancer and rare diseases. This project will be run by Genomics England, a company established in July 2013 by the UK Department of Health, together with Illumina for sequencing and data analysis pipelines instruments and infrastructure provider. They selected four companies in July 2015 to work on interpreting genomic data from the first 8,000 patients participating in the project: WuXi NextCODE for interpreting variants found in individuals with both cancer and rare diseases, Congenica and Omicia for rare-disease interpretation, and NanHealth for oncology. The study will last 3 years; if it is successful, Illumina anticipates that it will lead to an expansion of the effort to sequence a greater proportion of the UK population.(92)

Lots of passionate scientists with their own interest enrich the continuity progress in science with diverse sparks, discoveries, or even disruptive. One interesting disruptive technology is the capillary sequencing, also known as Sanger sequencing.(93) It enabled the initial sequencing of the human genome $(55,94)$ and led to the second Noble prize for the late Dr. Fred Sanger in 1980 (http://www.nobelprize.org/ nobel_prizes/chemistry/laureates/1980/). This technology is simple and elegant, ushered in the dawning of the most successful years in cardiovascular genetics and deciphering the genetic basis of single gene cardiovascular disorders, for example hereditary cardiomyopathies, ion channel disorders, and autosomal-dominant hypercholesterolemia, among others.(95-99)

Application of the massively parallel sequencing technology to genetic testing at the clinic, however, exposed even bigger challenges of how to interpret the findings 
Table 1. NGS methods.(92) (Adapted with permission from Nature).

PRECISION MEDICINE TOOLBOX
NGS platforms can answer questions related not only to the exome or genome but also the
transcriptome and epigenome of any organism. Sequencing methods differ in terms of how samples
are obtain and the data analysis involved.
WGS
WGS detects the 3.2 billion bases of the human genome. The ability to sequence large cohorts is now
a reality, and WGS will enable deeper understanding of the regulatory and other features in the human
genome, as well as meaningful interpretations of whole genomes. WGS is also important for
agriculture and microbial genomes.
De novo sequencing
This method refers to sequencing of a novel genome for which there is no available reference
sequence for alignment. The quality of the data depends on the size and continuity of the gaps in the
data.
WES
WES captures only the protein coding part of the genome. Representing less than $2 \%$ of the human
genome, WES is a cost-effective alternative to WGS. It is used for many applications, including
investigating genetic disease, population genetics and cancer studies.
Transcriptome sequencing
This method creates a biological snapshot of expressed genes by capturing RNA and converting it to
cDNA before sequencing. RNA sequencing can focus on mRNA, small RNA, noncoding RNA or
microRNA, depending on the steps included befire cDNA synthesis.

Epigenome sequencing

Epigenome sequencing investigates heritable changes in gene activity caused by environmental factors, such as DNA methylation and acetylation, DNA-protein interactions, small RNA-mediated regulation and histone modifications.

and apply them to the care of the individual. The primary challenge has been identifying the causal variant(s) among 4 million or so variants in each genome, including $>12,000$ non-synonymous, and several hundred if not thousand putatively functional and pathogenic variants. $(100,101)$ The plethora of the variants in each genome is inherent to the rare error rate of DNA replication machinery, which is estimated as well as empirically shown to be at $1 \times 10^{-8}$ per base. $(102,103)$ Accordingly, $\approx 30$ de novo variants are generated with each meiosis. The explosive growth of human population during the past millennium really presented an enormous challenge in human genetic diversity, as each birth contribute $\approx 30$ new variants to the population genetic pool.(104) Variants with a minor allele frequency of $<0.01$, known as rare variants, are more common to be found in the population, compare to the common variants.(105) NGS systems are typically represented by SOLiD/Ion Torrent PGM from Life Sciences, Genome Analyzer/HiSeq 2000/ MiSeq from Illumina, and GS FLX Titanium/GS Junior from Roche. Wide application of this technology makes it possible to aid the achievement of goals to decode life mysteries, make better crops, detect pathogens, and improve life qualities.(106)
DNA sequencing has evolved from Maxam-Gilbert and Sanger methods in the 1970s to a set of technologies that are collectively referred to as NGS.(93,107-117) NGS sequences millions of short fragments of DNA in parallel, while the first generation perform just one DNA fragment at a time. Sequencing of DNA as a clinical test became routinely possible only after the automation of Sanger sequencing methods introduced in the mid-1990s, which used capillary gel electrophoresis with fluorescence-based detection. $(118,119)$ The throughput of NGS far surpasses that of automated Sanger sequencing. The higher throughput and lower per-base cost of NGS have contributed to its rapid adoption in clinical testing (120), despite the fact that several aspects of NGS analysis have much higher complexity.

The NGS consolidates two processes: the analytic wet bench process and bioinformatics analysis of sequence data. The first component generally includes any or all of the following processes: patient samples handling, nucleic acids extraction, fragmentation, patient samples barcoding (molecular indexing), enrichment of targets for exome or gene panels, adapter ligation, amplification, library preparation, flow cell loading, and generation of sequence reads. Sequence generation is almost entirely automated and 
the output consists of millions to billions of short sequence reads. The wet bench workflow is followed by intensive computational and bioinformatics analyses with application of variety of algorithms to map and align the short sequence reads to a linear reference human genome sequence. After mapping and alignment, variant calls are made at locations where nucleotides differ from the reference sequence. Due to the content needed, several separate processes then developed to analyze the clinical relevance of variants, either singly or in combination, related to their contribution to a given clinical phenotype.(121)

The College of American Pathologist (CAP) NGS Work Group approached the analytic wet bench process and the bioinformatics or "dry bench" analyses as 2 discrete processes requiring separate considerations for standards. The principles and guidelines (Supplementary Guidelines) developed by the Next-Generation Sequencing: Standardization of Clinical Testing (Nex-StoCT) workgroup. They represent the initial steps to perform a reliable and useful NGS-based test results related to clinical decision making. There are four components of quality management in clinical environment addressed in this guidelines: test validation, quality control procedures to assure and maintain accurate test results, the independent assessment of test performance through proficiency testing or alternative approaches and reference materials.(122)

The translation of NGS from basic to clinical research and adoption for clinical diagnostics has occurred over a relatively short period of time. A growing number of clinical laboratories are implementing NGS-based diagnostic assays, mostly in the form of multigene panels, although an increasing number of laboratories are performing exome and genome sequencing. CAP identified that the adoption of NGS by clinical laboratories required the development of accreditation requirements specific to NGS. To assist clinical laboratories with the validation of NGS methods and platforms, the ongoing monitoring of NGS testing to ensure quality results, and the interpretation and reporting of variants found using these technologies, the American College of Medical Genetics and Genomics has developed the following professional standards and guidelines.(123)

\section{Bioinformatics}

We are on the verge of the genomic era: doctors and patients will have access to genetic data to customize medical treatment. Consumers can already get 500,000$1,000,000$ variant markers analyzed with associated trait information (124), and soon full genome sequencing will cost less than $\$ 1,000$ (125). One group has performed a complete clinical assessment of a patient using a personal genome (126), and the 1,000 Genomes Project is sequencing 1,000 individuals (63). In the coming years, individual genomic data will inundate the bioinformatics world, and these will set other challenges the bioinformatics community needs to address. In the last decade, molecular science has made many advances to benefit medicine, including the Human Genome project, International HapMap project and genome wide association studies.(64) Single nucleotide polymorphisms (SNPs) are now recognized as the main cause of human genetic variability and are already a valuable resource for mapping complex genetic traits.(127) Thousands of DNA variants have been identified that are associated with diseases and traits.(124) By combining these genetic associations with phenotypes and drug response, personalized medicine will tailor treatments to the patients' specific genotype (Figure 6).(40)

Precision medicine associate the detailed, patientspecific molecular information to diagnose and categorize disease, as a treatment guide to improve clinical outcome.(6) In precision medicine, it is assumed that the underlying molecular causes of disease are at least partly specific to each patient, that is, each patient has a unique set of molecular alterations that are responsible for their disease condition. Identifying these molecular alterations

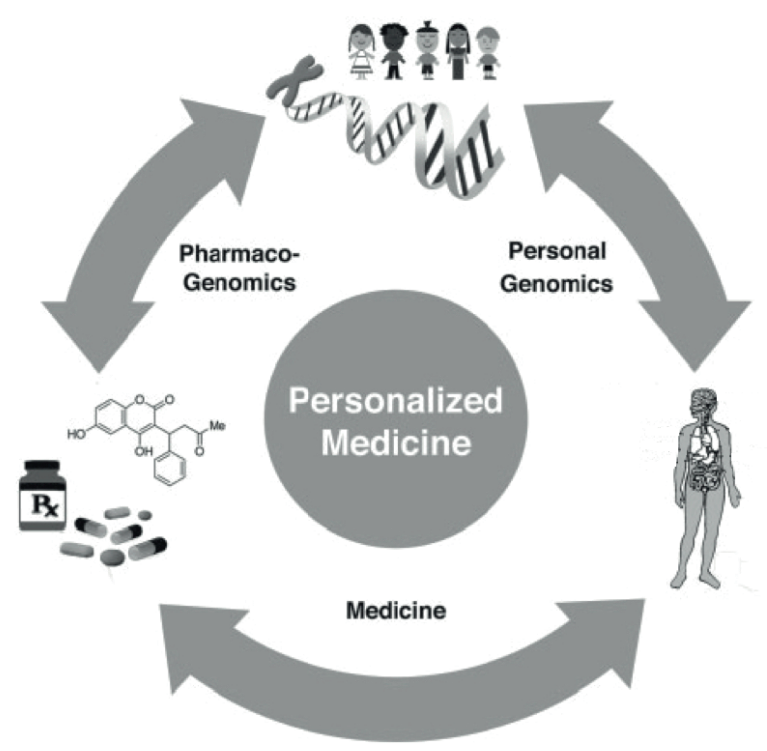

Figure 6. Personalized medicine. Personal genomics connect genotype to phenotype and provide insight into disease. Pharmacogenomics connect genotype to patient-specific treatment.(40) (Adapted with permission from Oxford University Press). 
helps identify the best treatment for each individual, thus effectively tailoring and customizing treatment for each individual. In some diseases, precision medicine relies on molecular biomarkers, that is, molecular events that are correlated with treatment response and clinical outcome but not necessarily causal for the disease.(128)

Currently, almost all precision medicine programs rely on NGS for patient's DNA sample examination, range from highly focused to specific regions (a few genes are sequenced), to whole-exome (all genes are sequenced) and whole-genome (the entire genome is sequenced). The ability to interrogate a large number of genes is clinically relevant because predicting the efficacy of a growing number of drugs requires knowledge of more than one molecular alteration.(129) A frequently encountered hurdle in the implementation of a precision medicine program is the bioinformatics and informatics component required to support such a program.(130) Indeed, informatics plays a key role in nearly every aspect of a precision medicine program, ranging from physician-oriented clinical interfaces that enable them to order tests and visualize and interpret results for decision support and report generation, to systems for sample tracking and handling, data acquisition, and software for analyzing the genomic assays including identification and annotation of variants.(129)

Bioinformatics is often defined as the application of computational techniques to understand and organize the information represents biological macromolecules. This unexpected union between the two subjects is largely attributed to the fact that life itself is an information technology; an organism's physiology is largely determined by its genes, which at its most basic can be viewed as digital information. At the same time, there have been major advances in the technologies that supply the initial data.(131) The aims of bioinformatics are three-fold. First, at its simplest one, bioinformatics organizes data in a way that researchers can easily access existing information or submit new data entries, e.g., the Protein Data Bank for 3D macromolecular structures.(132,133) While data-curation is an essential task, the information stored in these databases is essentially useless until analyzed. Thus the purpose of bioinformatics extends much further. The second aim is developing tools and resources to perform faster data analysis. For example, sequencing a particular protein, or compare it with previously characterized sequences. This needs more than just a simple text-based search and programs such as FASTA format (134) and Position-Specific Iterative Basic Local Alignment Search Tool (PSI-BLAST) (135) to consider what comprises a biologically significant match. Development of such resources required expertise in computational theory as well as a thorough understanding of biology. The third aim of these tools is to analyze the data and interpret the results in a biologically meaningful manner. Traditional biological studies examined individual systems in detail, then oftenly compared them with a few that are related. In bioinformatics, we now can perform a global analysis of all the available data and uncover common principles that apply across many systems and highlight novel features.(131)

It may now cost less to sequence the three billion DNA base pairs of a human genome than to do a brain scan. But how to translate all that genomic data into treatment? The resulting era of "precision medicines" is already delivering treatments tailored to individual needs. These 'big data' efforts face huge challenges, from creating analytic tools and solving scientific puzzles to accessing millions of gigabytes of data and overcoming barriers to accessing patients' health records.(136) Further advances in bioinformatics combined with experimental genomics for individuals are predicted to revolutionize the future of health care.

\section{Implementing Personalized Medicine into Medical Practice}

The completion of the first human genome sequence in 2003 created much anticipation and promise among scientists, health care providers, media, and the public.(137) However, this did not result immediately in tangible changes in standard medical care. While the media continued to anticipate and the public keep waiting, genomic research progressed. Over the last few years, impressive strides have been made to this effect. In recent years, emerging evidence suggests a rapidly growing expectation to incorporate genomic medicine into individualized patient care.(138) The promise of genomic medicine, as one part of individualized care, is to enable medical practitioners to make better clinical decisions through an improved informed process. The anticipated results are to improve targeted therapies, reduce side-effects, increase prevention and prediction of disease, enable earlier disease intervention, reduce healthcare costs and improve patient outcomes.(139) A human 'cancer genome', or oncogenome, is a residential for numerous chromosomes, chromatin (the fibers that constitute the chromosomes) and nucleotides alterations. These include irreversible aberrations in the sequence or structures of DNA, genes or chromosomes (that is, the copy number of the DNA). They could also include potentially 
reversible changes, known as epigenetic modifications to the DNA and/or to the histone proteins, which are closely associated with the DNA in chromatin. These reversible and irreversible changes affect hundreds to thousands of genes and/or regulatory transcripts. Collectively, they result in the activation or inhibition of various biological events, thereby causing aspects of cancer pathophysiology, including angiogenesis, immune evasion, metastasis, and altered cell growth, death and metabolism.(140,141) The baseline information about frequent genomic alterations in cancer generated in the research setting by sequencing the DNA of thousands of tumors is now being coupled with NGSbased methods that rapidly generate the mutational profile of a cancer genome in the clinical setting to inform genomeguided cancer medicine.(142)

Insights into the molecular pathology of disease are creating opportunities for the development of therapies with durable clinical benefit while challenging existing model of therapeutic development and clinical care.(141,143,144) Large international consortia, such as the ICGC $(145,146)$, are mapping the genomes of thousands of cancers to identify opportunities for prevention, early detection and treatment (147). Although genomics is leading the way, highthroughput proteomics and metabolomics are following closely behind.(148) These methodological advances have ushered in a target specific molecular processes new era of therapeutics. Though there have been some successes, the overall strategy remains in its infancy.(149-159) The central premise of precision medicine is that matching a drug and its mechanism of action using a marker to select patients, a process often referred to as matching the right drug to the right patient, can offer greater potential for durable clinical benefits.(160)

Metabolomics allows for a global assessment of a cellular state within the context of the immediate environment, taking into account genetic regulation, altered kinetic activity of enzymes, and changes in metabolic reactions.(161-163) Thus, compared with genomics or proteomics, metabolomics reflects changes in phenotype and therefore function. The omic sciences are, however, complementary as "upstream" changes in genes and proteins are measured "downstream" as changes in cellular metabolism.(161,164). Other features of metabolomics are similar to those of proteomics and transcriptomics, including the ability to assay biofluids or tumor samples and the relatively inexpensive, rapid, and automated techniques once start-up costs are taken into account.(165)

Personalized approaches reach the full spectrum of cancer care. Personalized risk assessment can provide patients identification at greatest risk of developing specific cancers, so they can be offered more comprehensive screening and prevention strategies, which will lead to fewer cases of invasive cancer, earlier diagnoses, and improved outcomes.(166) Personalized medicine has potential to change the standard of care for cardiovascular diseases. Although there is only a few examples of personalized cardiovascular medicine based on molecular profiling exist to date, while other methods have been used. Certainly, normalization of drug exposure across different subsets of individuals is one form of personalized medicine that is well established. The development of personalized medicine strategies based on genetic or physiological biomarkers for cardiovascular diseases such as atherosclerosis, heart failure and hypertension is challenging because of the multifactorial etiology of these diseases.(4)

Cardiovascular diseases originate from the confluence of many different factors. Genetic factor plays only a weak effect on the process taken as a whole, but it may substantially influence one of the known underlying pathways. For example, genetic effects on lipid biomarkers may often be more readily detected than their effect on myocardial infarction.(101) Genetic linkage analysis in large families, which led to deciphering the molecular genetic basis of single gene disorders, such as hereditary cardiomyopathies and ion channel disorders $(95,97,168,169)$, continues to offer a robust platform for identification of the causal genes for single gene disorders. Signal-transducing adaptor protein 1 (STAP1), encoding signal transducing adaptor family member 1, was mapped recently as a novel gene for autosomal dominant familial hypercholesterolemia through linkage analysis.(169) Further characterization of the locus after exome sequencing and showing evidence of enrichment of STAP1 variants in an independent cohort with familial hypercholesterolemia supported the causal role of STAP1 in autosomal dominant familial hypercholesterolemia. Thus, STAP1 joins the previously identified low-density lipoprotein receptor (LDLR), apolipoprotein B (APOB), and proprotein convertase subtilisin/kexin type 9(PCSK9) genes, as the fourth causal gene associated with this rare single gene disorder.(98,170,171) The genetic cardiomyopathies present a window to cardiac pathophysiology when discrete cellular pathways are disrupted. Over the past decades, the role of numerous proteins in triggering cardiomyopathy and hence HF has finally become clear. Despite the genetic complexity, direct application of genetic testing is now a mainstay in managing affected families, and scientifically and clinically useful themes are emerging that should lead to improved treatment.(95) 
Investigations of rare monogenic disorders of heart rhythm has elucidated the fundamental molecular and genetic mechanisms of sickle cell disease. After identification of more than 25 causal genes, there remain many subjects with inherited arrhythmia susceptibility but do not have mutations, this suggests that there is still other genes left unidentified. Newer strategies such as exome and WGS may be valuable to uncover additional molecular etiologies. Efforts to understand mechanisms responsible for incomplete penetrance, including identification of modifier genes, will also contribute to deciphering the complex relationships between genotype and phenotype.(97)

In diabetes, personalized medicine refers to utilize the patients specific characters for most effective diagnostic or treatment strategies. These include individual behavioral and phenotypic features, standard clinical laboratory findings, and gene sequences and other molecular markers.(172) Diabetes mellitus has long been recognized to be a complex, heterogeneous disorder, especially in type 2 diabetes patients with substantial variability in genetic risk factors, underlying pathogenic mechanisms, and clinical features. Therefore it represents a human disease that gains a substantial benefit from personalized approaches to treatment. Nevertheless, patients with type 2 diabetes often are treated similarly, with little consideration of individual characteristics that might affect clinical outcome and therapeutic response.(173) Both type 1 and type 2 diabetes are thought to be complex diseases, which means they need the interplay of numerous susceptibility and protective genes, acting in concert with negative and positive environmental factors to be developed.(174)

Type 2 diabetes typically is characterized by a combination of abnormalities in both insulin secretion and responsiveness, plus a more gradual and less extensive loss of $\beta$-cell secretory capacity than occurs in type 1 diabetes. For this reason, a spectrum of pharmacologic agents with actions that include augmentation of insulin sensitivity, stimulation of insulin secretion, and slowing of intestinal glucose absorption. Should be the available options for glycemic management in type 2 diabetes and not only exogenous insulin. $(173,174)$ The application of systems biology methods to complex diseases such as diabetes mellitus is now being explored as a strategy for amplifying insights into pathophysiology and disease management by integrating the expanding amount of molecular data. $(175,176)$ It is likely that personalized medicine in more common forms of diabetes can have substantial benefit by similarly using individual patient characteristics to define a preferred sequence of options in treatment rather than one specific therapy.(172) New technology in human genetics transformation become the single best hope to innovate and improve clinical success rates in drug development.

\section{Conclusion}

Technologies for monitoring individuals' health are becoming increasingly available, especially with consumer electronic devices moving into health measurements. The devices currently measure mostly vital signs, but it is inevitable they will move into blood tests and portable imaging in the future. The real ambition of personalized medicine said Goldstein, "is in transforming the way we develop new medicines." He also believes that "other technological drivers will be in genome editing and stem cell biology, since they together create a clear pathway for in vitro models of many human diseases." It is this deep appreciation for the unique genetic and phenotypic characteristics of an individual that is elegantly depicted by Sir William Osler famous quote: The good physician treats the disease, the great physician treats the patient who has the disease.

\section{References}

1. Ginsburg GS, Willard HF. Genomic and personalized medicine: foundations and applications. Transl Res. 2009; 154: 277-87.

2. Topol EJ. Individualized medicine from prewomb to tomb. Cell. 2014; 157: 241-53.

3. FDA. Paving the Way for Personalized Medicine: FDA's Role in a New Era of Medical Product Development. Maryland: U.S. Food and Drug Administration; 2013.

4. Blaus A, Madabushi R, Pacanoski M, Rose M, Schuck RN, Stockbridge N, et al. Personalized cardiovascular medicine today: a Food and Administration/Center for Drug evaluation and research perspective. Circulation. 2015; 132: 1425-32.

5. The White House [Internet]. Fact Sheet: President Obama's Precision Medicine Initiative [update 2015 Jan 30, cited 2016 Jul 29]. Available from: https://www.whitehouse.gov/the-press-office/2015/01/30/ fact-sheet-president-obama-s-precision-medicine-initiative.

6. National Research Council. Toward Precision Medicine: Building a Knowledge Network for Biomedical Research and a New Taxonomy of Disease. Washington DC: The National Academies Press; 2011.

7. Aronson SJ, Rehm HL. Building the foundation for genomics in precision medicine. Nature. 2015; 526: 336-42.

8. Relling MV, Gardner EE, Sandborn WJ, Schmiegelow K, Pui CH, Yee $\mathrm{SW}$, et al. Clinical pharmacogenetics implementation consortium guidelines for thiopurine methyltransferase genotype and thiopurine dosing: 2013 update. Clin Pharmacol Ther. 2013; 93: 324-5.

9. Korf BR, Rehm HL. New approaches to molecular diagnosis. J Am Med Assoc. 2013; 309: 1511-21. 
10. Hoffman MA, Williams MS. Electronic medical records and personalized medicine. Hum Genet. 2011; 130: 33-9.

11. Del Fiol G. Williams MS, Maram N, Rocha RA, Wood GM, Mitchell JA. Integrating genetic information resources with an EHR. AMIA Annu Symp Proc. 2006; 2006: 904.

12. Béroud C, Collod-Béroud G, Boileau C, Soussi T, Junien C. UMD (Universal mutation database): a generic software to build and analyze locus-specific databases. Hum Mutat. 2000; 15: 86-94.

13. Sosnay PR, Siklosi KR, Van Goor F, Kaniecki K, Yu H, Sharma N, et al. Defining the disease liability of variants in the cystic fibrosis transmembrane conductance regulator gene. Nature Genet. 2013; 45: 1160-7.

14. Firth HV, Richards SM, Bevan AP, Clayton S, Corpas M, Rajan D, et al. DECIPHER: Database of chromosomal imbalance and phenotype in humans using ensembl resources. Am J Hum Genet. 2009; 84: 524-33.

15. Miller DT, Adam MP, Aradhya S, Biesecker LG, Brothman AR, Carter NP, et al. Consensus statement: chromosomal microarray is a first-tier clinical diagnostic test for individuals with developmental disabilities or congenital anomalies. Am J Hum Genet. 2010; 86: 749-64.

16. Thompson BA, Spurdle AB, Plazzer JP, Greenblatt MS, Akagi K, A1Mulla F, et al. Application of a 5-tiered scheme for standardized classification of 2,360 unique mismatch repair gene variants in the InSiGHT locus-specific database. Nature Genet. 2014; 46: 107-15.

17. Tryka KA, Hao L, Sturcke A, Jin Y, Wang ZY, Ziyabari L, et al. NCBI's Database of Genotypes and Phenotypes: dbGaP. Nucleic Acids Res. 2014; 42: D975-9.

18. Zhang J, Baran J, Cros A, Guberman JM, Haider S, Hsu J, et al. International Cancer Genome Consortium Data Portal -- a one-stop shop for cancer genomics data. Database (Oxford). 2011; 2011: bar026. doi: 10.1093/database/bar026.

19. The Cancer Genome Atlas Research Network, Weinstein JN, Collisson EA, Mills GB, Shaw KR, Ozenberger BA, et al. The Cancer Genome Atlas Pan-Cancer analysis project. Nature Genet. 2013; 45: 1113-20.

20. Schilsky RL, Michels DL, Kearbey AH, Yu PP, Hudis CA. Building a rapid learning health care system for oncology: the regulatory framework of CancerLinQ. J Clin Oncol. 2014; 32: 2373-9.

21. Nicholson JK. Global systems biology, personalized medicine and molecular epidemiology. Mol Sys Biol. 2006; 2: 52.

22. MacArthur DG, Balasubramanian S, Frankish A, Huang N, Morris J, Walter K, et al. A Systematic survey of loss-of-function variants in human protein-coding genes. Science. 2012; 335: 823-8.

23. Ahrens CH, Brunner E, Qeli E, Basler K, Aebersold R. Generating and navigating proteome maps using mass spectrometry. Nature Rev Mol Cell Biol. 2010; 11: 789-801.

24. Bensimon A, Heck AJ, Aebersold R. Mass spectrometry-based proteomics and network biology. Annu Rev Biochem. 2012; 81: 379-405.

25. Richards AL, Merrill AE, Coon JJ. Proteome sequencing goes deep. Curr Opin Chem Biol. 2015; 24: 11-7.

26. Walther TC, Mann M. Mass spectrometry-based proteomics in cell biology. J Cell Biol. 2010; 190: 491-500.

27. Larance M, Lamond AI. Multidimensional proteomics for cell biology. Nat Rev Mol Cell Biol. 2015; 16: 269-80.

28. Nicholson JK, Connelly J, Lindon JC, Holmes E. Metabonomics: a platform for studying drug toxicity and gene function. Nat Rev Drug Discov. 2002; 1: 153-61.

29. Clish CB. Metabolomics: an emerging but powerful tool for precision medicine. Cold Spring Harb Mol Case Stud. 2015; 1: a000588. doi: 10.1101/mcs.a000588.
30. Kuehnbaum NL, Britz-McKibbin P. New advances in separation science for metabolomics: resolving chemical diversity in a postgenomic era. Chem Rev. 2013; 113: 2437-68.

31. Gieger C, Geistlinger L, Altmaier E, de Angelis MH, Kronenberg F, Meitinger T, et al. Genetics meets metabolomics: a genome-wide association study of metabolite profiles in human serum. Plos Gen. 2008; 4: e1000282. doi: 10.1371/journal.pgen.1000282.

32. Wishart DS, Jewison T, Guo AC, Wilson M, Knox C, Liu Y, et al. HMDB 3.0 -- the human metabolome database in 2013. Nucleic Acids Res. 2013; 41: D801-7.

33. Mayers JR, Wu C, Clish CB, Kraft P, Torrence ME, Fiske BP, et al. Elevation of circulating branched-chain amino acids is an early event in human pancreatic adenocarcinoma development. Nat Med. 2014; 20: 1193-8.

34. Rhee EP, Cheng S, Larson MG, Walford GA, Lewis GD, McCabe E, et al. Lipid profiling identifies a triacylglycerol signature of insulin resistance and improves diabetes prediction in humans. J Clin Invest. 2011; 121: 1402-11.

35. Wang TJ, Larson MG, Vasan RS, Cheng S, Rhee EP, McCabe E, et al. Metabolite profiles and the risk of developing diabetes. Nat Med. 2011; 17: 448-53.

36. Wang TJ, Ngo D, Psychogios N, Dejam A, Larson MG, Vasan RS, et al. 2-aminoadipic acid is a biomarker for diabetes risk. J Clin Invest. 2013; 123: 4309-17.

37. Mapstone M, Cheema AK, Fiandaca MS, Zhong X, Mhyre TR, MacArthur LH, et al. Plasma phospholipids identify antecedent memory impairment in older adults. Nat Med. 2014; 20: 415-8.

38. Newgard CB, An J, Bain JR, Muehlbauer MJ, Stevens RD, Lien LF, et al. A branched-chain amino acid-related metabolic signature that differentiates obese and lean humans and contributes to insulin resistance. Cell Metab. 2009; 9: 311-26.

39. Baynam G, Walters M, Claes P, Kung S, LeSouef P, Dawkins H, et al. Phenotyping: targeting genotype's rich cousin for diagnosis. J Paediatr Child Health. 2015; 51: 381-6.

40. Fernald GH, Capriotti E, Daneshjou R, Karczewski KJ, Altman RB. Bioinformatics challenges for personalized medicine. Bioinformatics. 2011; 27: 1741-8.

41. Robinson PN, Mungall CJ, Haendel M. Capturing phenotypes for precision medicine. Cold Spring Harb Mol Case Stud. 2015; 1: a000372. doi: 10.1101/mcs.a000372.

42. Robinson PN. Deep phenotyping for precision medicine. Hum Mutat. 2012; 33: 777-80.

43. Riggs ER, Jackson L, Miller DT, Van Vooren S. Phenotypic information in genomic variant databases enhances clinical care and research: the International Standards for Cytogenomic Arrays Consortium experience. Hum Mutat. 2012; 33: 787-96.

44. Thakuria JV, Zaranek AW, Church GM, Berry GT. Back to the future: from genome to metabolome. Hum Mutat. 2012; 33: 809-12.

45. Taboada M, Rodríguez H, Martínez D, Pardo M, Sobrido MJ. Automated semantic annotation of rare disease cases: a case study. Database (Oxford). 2014; 2014 pii: bau045. doi: 10.1093/database/ bau045.

46. Kuleshov V, Xie D, Chen R, Pushkarev D, Ma Z, Blauwkamp T, et al. Whole-genome haplotyping using long reads and statistical methods. Nat Biotechnol. 2014; 32: 261-6.

47. Welch KO, Marin RS, Pandya A, Arnos KS. Compound heterozygosity for dominant and recessive GJB2 mutations: effect on phenotype and review of the literature. Am J Med Genet A. 2007; 143A: 1567-73.

48. Fong CYI, Mumford AD, Likeman MJ, Jardine PE. Cerebral palsy in siblings caused by compound heterozygous mutations in the gene encoding protein C. Dev Med Child Neurol. 2010; 52: 489-93. 
49. Shimizu H, Takizawa Y, Pulkkinen L, Murata S, Kawai M, Hachisuka $\mathrm{H}$, et al. Epidermolysis bullosa simplex associated with muscular dystrophy: phenotype-genotype correlations and review of the literature. J Am Acad Dermatol. 1999; 41: 950-6.

50. Tewhey R, Bansal V, Torkamani A, Topol EJ, Schork NJ. The importance of phase information for human genomics. Nat Rev Genet. 2011; 12: 215-23.

51. Browning SR, Browning BL. Haplotype phasing: existing methods and new developments. Nat Rev Genet. 2011; 12: 703-14.

52. Kitzman JO, Mackenzie AP, Adey A, Hiatt JB, Patwardhan RP, Sudmant $\mathrm{PH}$, et al. Haplotype-resolved genome sequencing of a Gujarati Indian individual. Nat Biotechnol. 2011; 29: 59-63.

53. Fan HC, Wang J, Potanina A, Quake SR. Whole-genome molecular haplotyping of single cells. Nat Biotechnol. 2011; 29: 51-7.

54. Green RE, Krause J, Briggs AW, Maricic T, Stenzel U, Kircher M, et al. A draft sequence of the Neandertal genome. Science. 2010; 328: $710-22$.

55. Lander ES, Linton LM, Birren B, Nusbaum C, Zody MC, Baldwin J, et al. Initial sequencing and analysis of the human genome. Nature. 2001; 409: 860-921.

56. Li R, Li Y, Fang X, Yang H, Wang J, Kristiansen K, et al. SNP detection for massively parallel whole-genome resequencing. Genome Res. 2009; 19: 1124-32.

57. Li H, Durbin R. Fast and accurate short read alignment with BurrowsWheeler transform. Bioinformatics. 2009; 25: 1754-60.

58. DePristo MA, Banks E, Poplin R, Garimella KV, Maguire JR, Hartl $\mathrm{C}$, et al. A framework for variation discovery and genotyping using next-generation DNA sequencing data. Nat Genet. 2011; 43: 491-8.

59. Levy S, Sutton G, Ng PC, Feuk L, Halpern AL, Walenz BP, et al. The diploid genome sequence of an individual human. PLoS Biol. 2007; 5: e254. doi: 10.1371/journal.pbio.0050254.

60. Wang J, Wang W, Li R, Li Y, Tian G, Goodman L, et al. The diploid genome sequence of an Asian individual. Nature. 2008; 456: 60-5.

61. Ahn SM, Kim TH, Lee S, Kim D, Ghang H, Kim DS, et al. The first Korean genome sequence and analysis: full genome sequencing for a socio-ethnic group. Genome Res. 2009; 19: 1622-9.

62. Tong P, Prendergast JG, Lohan AJ, Farrington SM, Cronin S, Friel N, et al. Sequencing and analysis of an Irish human genome. Genome Biol. 2010; 11: R91.

63. 1000 Genomes Project Consortium, Abecasis GR, Altshuler D, Auton A, Brooks LD, Durbin RM, et al. A map of human genome variation from population-scale sequencing. Nature. 2010; 467: 1061-73.

64. International HapMap Consortium. A haplotype map of the human genome. Nature. 2005; 437: 1299-320.

65. 1000 Genomes Project Consortium, Abecasis GR, Auton A, Brooks LD, DePristo MA, Durbin RM, et al. An integrated map of genetic variation from 1,092 human genomes. Nature. 2012; 491: 56-65.

66. Cao H, Wu H, Luo R, Huang S, Sun Y, Tong X, et al. De Novo assembly of a haplotype-resolved human genome. Nat Biotechnol. 2015; 33: 617-22.

67. Browning SR, Browning BL. Rapid and accurate haplotype phasing and missing-data inference for whole-genome association studies by use of localized haplotype clustering. Am J Hum Genet. 2007; 81: 1084-97.

68. Duitama J, McEwen GK, Huebsch T, Palczewski S, Schulz S, Verstrepen $\mathrm{K}$, et al. Fosmid-based whole genome haplotyping of a HapMap trio child: evaluation of single individual haplotyping techniques. Nucleic Acids Res. 2012; 40: 2041-53.

69. Woolley AT, Guillemette C, Li Cheung C, Housman DE, Lieber CM. Direct haplotyping of kilobase-size DNA using carbon nanotube probes. Nat Biotechnol. 2000; 18: 760-3.
70. Cao H, Wang Y, Zhang W, Chai X, Zhang X, Chen S, et al. A shortread multiplex sequencing method for reliable, cost-effective and high-throughput genotyping in large-scale studies. Hum Mutat. 2013; 34: 1715-20.

71. Dapprich J, Ferriola D, Magira EE, Kunkel M, Monos D. SNPspecific extraction of haplotype-resolved targeted genomic regions. Nucleic Acids Res. 2008; 36: e94. doi: 10.1093/nar/gkn345.

72. Suk EK, McEwen GK, Duitama J, Nowick K, Schulz S, Palczewski $\mathrm{S}$, et al. A comprehensively molecular haplotype-resolved genome of a European individual. Genome Res. 2011;21: 1672-85.

73. ABPI. The stratification of disease for personalised medicines: Research driven recommendations to strengthen a unified UK strategy through a stakeholder alliance. London: The Association of the British Pharmaceutical Industry; 2014.

74. [N.A.]. The coming era of human phenotyping. Nat Biotechnol. 2015; 6: 567. doi: 10.1038/nbt.3266.

75. Relling MV, Evans WE. Pharmacogenomic in the clinic. Nature. 2015; 526: 343-50.

76. Kinch MS, Haynesworth A, Kinch SL, Hoyer D. An overview of FDA-approved new molecular entities: 1827-2013. Drug Discov Today. 2014; 19: 1033-9.

77. Ehmann F, Caneva L, Prasad K, Paulmichl M, Maliepaard M, Llerena A, et al. Pharmacogenomic information in drug labels: European Medicines Agency perspective. Pharmacogenomics J. 2015; 15: 201-10.

78. Ishiguro A, Yagi S, Uyama Y. Characteristics of pharmacogenomics/ biomarker-guided clinical trials for regulatory approval of anticancer drugs in Japan. J Hum Genet. 2013; 58: 313-6.

79. U.S. Food and Drug Administration [Internet]. Table of Pharmacogenomic Biomarkers in Drug Labeling [updated $2016 \mathrm{Jul}$ 11, cited 2016 Jul 29]. Available from: http:/www.fda.gov/drugs/ scienceresearch/researchareas/pharmacogenetics/ucm083378.htm.

80. CPIC (Clinical Pharmacogenetics Implementation Consortium) [Internet]. Genes - Drugs [updated 2016 May 13; cited $2016 \mathrm{Jul}$ 29]. Available from: https://cpicpgx.org/genes-drugs/.

81. Dunnenberger HM, Crews KR, Hoffman JM, Caudle KE, Broeckel $\mathrm{U}$, Howard SC, et al. Preemptive clinical pharmacogenetics implementation: current programs in five US medical centers. Annu Rev Pharmacol Toxicol. 2015; 55: 89-106.

82. Buchanan J, Wordsworth $\mathrm{S}$, Schuh A. Issues surrounding the health economic evaluation of genomic technologies. Pharmacogenomics. 2013; 14: 1833-47.

83. Schroeder SA, Frist W. Phasing out fee-for-service payment. N Engl J Med. 2013; 368: 2029-32.

84. Levy KD, Decker BS, Carpenter JS, Flockhart DA, Dexter PR, Desta Z, et al. Prerequisites to implementing a pharmacogenomics program in a large health-care system. Clin Pharmacol Ther. 2014; 96: 307-9.

85. Gordon AS, Tabor HK, Johnson AD, Snively BM, Assimes TL, Auer PL, et al. Quantifying rare, deleterious variation in 12 human cytochrome P450 drug-metabolism genes in a large-scale exome dataset. Hum Mol Genet. 2014; 23: 1957-63.

86. Chen Z, Liew D, Kwan P. Effects of a HLA-B*15:02 screening policy on antiepileptic drug use and severe skin reactions. Neurology. 2014; 83: 2077-84

87. Landrum MJ, Lee JM, Riley GR, Jang W, Rubinstein WS, Church DM, et al. ClinVar: public archive of relationships among sequence variation and human phenotype. Nucleic Acids Res. 2014; 42: D980-5.

88. Rehm HL, Berg JS, Brooks LD, Bustamante CD, Evans JP, Landrum MJ, et al. ClinGen -- the Clinical Genome Resource. N Engl J Med. 2015; 372: 2235-42. 
89. Whirl-Carrillo M, McDonagh EM, Hebert JM, Gong L, Sangkuhl K, Thorn CF, et al. Pharmacogenomics knowledge for personalized medicine. Clin Pharmacol Ther. 2012; 92: 414-7.

90. Carter RF. Adapting next generation technologies to clinical molecular oncology service. Clin Lab News. 2015; 2015: 22-8.

91. Dorschner MO, Amendola LM, Shirts BH, Kiedrowski L, Salama $\mathrm{J}$, Gordon AS, et al. Refining the structure and content of clinical genomic reports. Am J Med Genet C Semin Med Genet. 2014; 1: 85-92.

92. Brazil M. Genomics technologies march into new markets. Med Tech Deal Makers. P:M8. Available from: http://www.nature.com/ medtechdealmakers.

93. Sanger F, Nicklen S, Coulson AR. DNA sequencing with chainterminating inhibitors. Proc Natl Acad Sci USA. 1977; 74: 5463-7.

94. Venter JC, Adams MD, Myers EW, Li PW, Mural RJ, Sutton GG, et $a l$. The sequence of the human genome. Science. 2001; 291: 130451.

95. Cahill TJ, Ashrafian H, Watkins H. Genetic cardiomyopathies causing heart failure. Circ Res. 2013; 113: 660-75.

96. Abifadel M, Varret M, Rabès JP, Allard D, Ouguerram K, Devillers $\mathrm{M}$, et al. Mutations in PCSK9 cause autosomal dominant hypercholesterolemia. Nat Genet. 2003; 34: 154-6.

97. George AL Jr. Molecular and genetic basis of sudden cardiac death. J Clin Invest. 2013; 123: 75-83.

98. Marian AJ. Recent developments in cardiovascular genetics and genomics. Circ Res. 2014; 115: e11-7.

99. Lai YC, Potoka KC, Champion HC, Mora AL, Gladwin MT. Pulmonary arterial hypertension: the clinical syndrome. Circ Res. 2014; 115: 115-30.

100. Marian AJ. Medical DNA sequencing. Curr Opin Cardiol. 2011; 26: 175-80.

101. Marian AJ, Belmont J. Strategic approaches to unraveling genetic causes of cardiovascular diseases. Circ Res. 2011; 108: 1252-69.

102. Kong A, Frigge ML, Masson G, Besenbacher S, Sulem P, Magnusson $\mathrm{G}$, et al. Rate of de novo mutations and the importance of father's age to disease risk. Nature. 2012; 488: 471-5.

103. 1000 Genomes Project Consortium, Abecasis GR, Altshuler D, Auton A, Brooks LD, Durbin RM, at al. A map of human genome variation from population-scale sequencing. Nature. 2010; 467: 1061-73.

104. Keinan A, Clark AG. Recent explosive human population growth has resulted in an excess of rare genetic variants. Science. 2012; 336: 740-3.

105. Marian AJ. The bottleneck in genetic testing. Circ Res. 2015; 117: 586-8.

106. Liu L, Li Y, Li S, Hu N, He Y, Pong R, et al. Comparison of nextgeneration sequencing systems. J Biomed Biotechnol. 2012; 2012: 251364. doi: $10.1155 / 2012 / 251364$.

107. Maxam AM, Gilbert W. A new method for sequencing DNA. Proc Natl Acad Sci USA. 1977; 74: 560-4.

108. Sanger F, Coulson AR. A rapid method for determining sequences in DNA by primed synthesis with DNA polymerase. J Mol Biol. 1975; 94: 441-8.

109. Bentley DR, Balasubramanian S, Swerdlow HP, Smith GP, Milton J, Brown CG, et al. Accurate whole human genome sequencing using reversible terminator chemistry. Nature. 2008; 456: 53-9.

110. Mardis ER. The impact of next-generation sequencing technology on genetics. Trends Genet. 2008; 24: 133-41.

111. Mardis ER. Next-generation DNA sequencing methods. Annu Rev Genomics Hum Genet. 2008; 9: 387-402.

112. Margulies M, Egholm M, Altman WE, Attiya S, Bader JS, Bemben LA, et al. Genome sequencing in microfabricated high-density picolitre reactors. Nature. 2005; 437: 376-80.
113. Metzker ML. Sequencing technologies: the next generation. Nat Rev Genet. 2010; 11: 31-46.

114. Rothberg JM, Hinz W, Rearick TM, Schultz J, Mileski W, Davey $\mathrm{M}$, et al. An integrated semiconductor device enabling non-optical genome sequencing. Nature. 2011; 475: 348-52.

115. Schuster SC. Next-generation sequencing transforms today's biology Nat Methods. 2008; 5: 16-8.

116. Shendure J, Ji H. Next-generation DNA sequencing. Nat Biotechnol. 2008; 26: 1135-45.

117. Valouev A, Ichikawa J, Tonthat T, Stuart J, Ranade S, Peckham H, et al. A high-resolution, nucleosome position map of C. elegans reveals a lack of universal sequence-dictated positioning. Genome Res. 2008; 18: 1051-63.

118. Hutchison CA 3rd. DNA sequencing: bench to bedside and beyond. Nucleic Acids Res. 2007; 35: 6227-37.

119. Metzker ML. Emerging technologies in DNA sequencing. Genome Res. 2005; 15: 1767-76.

120. Mardis ER. A decade's perspective on DNA sequencing technology. Nature. 2011; 470: 198-203.

121. Aziz N, Zhao Q, Bry L, Driscoll D, Funke B, Gibson JS, et al. College of American Pathologists' laboratory standards for next-generation sequencing clinical tests. Arch Pathol Lab Med. 2015; 139: 481-93.

122. Gargis AS, Kalman L, Berry MW, Bick DP, Dimmock DP, Hambuch $\mathrm{T}$, et al. Assuring the quality of next-generation sequencing in clinical laboratory practice. Nat Biotechnol. 2012; 30: 1033-6.

123. Rehm HD, Bale SJ, Bayrak-Toydemir P, Berg JS, Brown KK, Deignan JL, et al. ACMG clinical laboratory standards for nextgeneration sequencing. Gen Med. 2013; 15: 733-47.

124. Hindorff LA, Sethupathy P, Junkins HA, Ramos EM, Mehta JP, Collins FS, et al. Potential etiologic and functional implications of genome-wide association loci for human diseases and traits. Proc Natl Acad Sci USA. 2009; 106: 9362-7.

125. Drmanac R, Sparks AB, Callow MJ, Halpern AL, Burns NL, Kermani $\mathrm{BG}$, et al. Human genome sequencing using unchained base reads on self-assembling DNA nanoarrays. Science. 2010; 327: 78-81.

126. Ashley EA, Butte AJ, Wheeler MT, Chen R, Klein TE, Dewey FE, et al. Clinical assessment incorporating a personal genome. Lancet. 2010; 375: 1525-35.

127. Collins FS, Guyer MS, Charkravarti A. Variations on a theme: cataloging human DNA sequence variation. Science. 1997; 278, 1580-1.

128. Keutgen XM, Filicori F, Crowley MJ, Wang Y, Scognamiglio T, Hoda $\mathrm{R}$, et al. A panel of four miRNAs accurately differentiates malignant from benign indeterminate thyroid lesions on fine needle aspiration. Clin Cancer Res. 2012; 18: 2032-8.

129. Sboner A, Elemento O. A primer on precision medicine informatics. Brief Bioinform. 2016; 17: 145-53

130. Servant N, Romejon J, Gestraud P, La Rosa P, Lucotte G, Lair S, et al. Bioinformatics for precision medicine in oncology: principles and application to the SHIVA clinical trial. Front Genet. 2014; 5: 152. doi: 10.3389/fgene.2014.00152.

131. Luscombe NM, Greenbaum D, Gerstein M. What is bioinformatics? An introduction and overview. Yearb Med Inform. 2001; 2001: 83100.

132. Bernstein FC, Koetzle TF, Williams GJ, Meyer EF Jr, Brice MD, Rodgers JR, et al. The Protein Data Bank. A computer-based archival file for macromolecular structures. Eur J Biochem. 1977; 80: 319-24.

133. Berman HM, Westbrook J, Feng Z, Gilliland G, Bhat TN, Weissig H, et al. The Protein Data Bank. Nucleic Acids Res. 2000; 28: 235-42.

134. Pearson WR, Lipman DJ. Improved tools for biological sequence comparison. Proc Natl Acad Sci USA. 1988; 85: 2444-8. 
135. Altschul SF, Madden TL, Schaffer AA, Zhang J, Zhang Z, Miller W, et al. Gapped BLAST and PSI-BLAST: a new generation of protein database search programs. Nucleic Acids Res. 1997; 25: 3389-402.

136. Bender E. Big data in biomedicine. Nature. 2015; 527: S1. doi: $10.1038 / 527$ s 1 a.

137. Green ED, Guyer MS, National Human Genome Research I. Charting a course for genomic medicine from base pairs to bedside. Nature. 2011; 470: 204-13.

138. Manolio TA, Chisholm RL, Ozenberger B, Roden DM, Williams MS, Wilson R, et al. Implementing genomic medicine in the clinic: The future is here. Genet Med. 2013; 15: 258-67.

139. Lazaridis KN, McAllister TM, Babovic-Vuksanovic D, Beck SA, Borad MJ, Bryce AH, et al. Implementing individualized medicine into the medical practice. Am J Med Gen C Semin Med Genet. 2014; 166C: $15-23$

140. Hanahan D, Weinberg RA. The hallmarks of cancer. Cell. 2000; 100: 57-70.

141. Hindorff LA, Sethupathy P, Junkins HA, Ramos EM, Mehta JP, Collins FS, et al. Potential etiologic and functional implications of genome-wide association loci for human diseases and traits. Proc Natl Acad Sci USA. 2009; 106: 9362-7.

142. Chin L, Gray JW. Translating insights from the cancer genome into clinical practice. Nature. 2008; 452: 553-63.

143. Andre F, Mardis E, Salm M, Soria JC, Siu LL, Swanton C. Prioritizing targets for precision cancer medicine. Ann Oncol. 2014; 25: 2295303.

144. Stratton MR. Exploring the genomes of cancer cells: progress and promise. Science. 2011; 331: 1553-8.

145. Stratton MR, Campbell PJ, Futreal PA. The cancer genome. Nature. 2009; 458: 719-24.

146. Hudson TJ, Anderson W, Artez A, Barker AD, Bell C, Bernabé RR, et al. International network of cancer genome projects. Nature. 2010; 464: 993-8. Erratum in: Nature. 2010; 465: 966.

147. The Cancer Genome Atlas Research Network. Comprehensive genomic characterization defines human glioblastoma genes and core pathways. Nature. 2008; 455: 1061-8. Erratum in: Nature. 2013; 494: 506

148. Chin L, Andersen JN, Futreal PA. Cancer genomics: from discovery science to personalized medicine. Nature Med. 2011; 17: 297-303.

149. Zhang B, Wang J, Wang X, Zhu J, Liu Q, Shi Z, et al. Proteogenomic characterization of human colon and rectal cancer. Nature. 2014; 513: 382-7.

150. Verweij J, Casali PG, Zalcberg J, LeCesne A, Reichardt P, Blay JY, et al. Progression-free survival in gastrointestinal stromal tumours with high-dose imatinib: randomised trial. Lancet. 2004; 364: 112734

151. Gerber DE, Minna JD. ALK inhibition for non-small cell lung cancer: from discovery to therapy in record time. Cancer Cell. 2010; 18: 548-51

152. Sosman JA, Kim KB, Schuchter L, Gonzalez R, Pavlick AC, Weber JS, et al. Survival in BRAF V600-mutant advanced melanoma treated with vemurafenib. N Engl J Med. 2012; 366: 707-14.

153. Slamon D, Eiermann W, Robert N, Pienkowski T, Martin M, Press M, et al. Adjuvant trastuzumab in HER2-positive breast cancer. N Engl J Med. 2011; 365: 1273-83

154. Shaw AT, Kim DW, Nakagawa K, Seto T, Crinó L, Ahn MJ, et al. Crizotinib versus chemotherapy in advanced ALK-positive lung cancer. N Engl J Med. 2013; 368: 2385-94.

155. Maemondo M, Inoue A, Kobayashi K, Sugawara S, Oizumi S, Isobe $\mathrm{H}$, et al. Gefitinib or chemotherapy for non-small-cell lung cancer with mutated EGFR. N Engl J Med. 2010; 362: 2380-8.
156. Ledermann J, Harter P, Gourley C, Friedlander M, Vergote I, Rustin $\mathrm{G}$, et al. Olaparib maintenance therapy in patients with platinumsensitive relapsed serous ovarian cancer: a preplanned retrospective analysis of outcomes by BRCA status in a randomised phase 2 trial. Lancet Oncol. 2014; 15: 852-61.

157. Kris MG, Johnson BE, Berry LD, Kwiatkowski DJ, Iafrate AJ, Wistuba II, et al. Using multiplexed assays of oncogenic drivers in lung cancers to select targeted drugs. J Am Med Assoc. 2014; 311: 1998-2006.

158. Jänne PA, Yang JC, Kim DW, Planchard D, Ohe Y, Ramalingam SS, et al. AZD9291 in EGFR inhibitor-resistant non-small-cell lung cancer. N Engl J Med. 2015; 372: 1689-99.

159. Demetri GD, von Mehren M, Blanke CD, Van den Abbeele AD, Eisenberg B, Roberts PJ, et al. Efficacy and safety of imatinib mesylate in advanced gastrointestinal stromal tumors. N Engl J Med. 2002; 347: 472-80.

160. Green ED, Guyer MS, National Human Genome Research Institute. Charting a course for genomic medicine from base pairs to bedside. Nature. 2011; 470: 204-13.

161. Blankin AV, Piantadosi S, Hollingsworth SI. Patient-centric trials for therapeutic development in precision oncology. Nature. 2015; 526: 361-70.

162. Griffin JL, Shockcor JP. Metabolic profiles of cancer cells. Nat Rev Cancer. 2004; 4: 551-61.

163. Mendes P, Kell DB, Westerhoff HV. Why and when channeling can decrease pool size at constant net flux in a simple dynamic channel. Biochim Biophys Acta. 1996; 1289: 175-86.

164. Mendes P, Kell DB, Westerhoff HV. Channelling can decrease pool size. Eur J Biochem. 1992; 204: 257-66.

165. Boros LG, Lerner MR, Morgan DL, Taylor SL, Smith BJ, Postier RG, et al. [1,2-13C2]-D-glucose profiles of the serum, liver, pancreas, and DMBA-induced pancreatic tumors of rats. Pancreas. 2005; 31: $337-43$.

166. Spratlin JL, Serkova NJ, Eckhardt SG. Clinical applications of metabolomics in oncology: a review. Clin Cancer Res. 2009; 15: 431-40.

167. Meric-Bernstam F, Farhangfar C, Mendelsohn J, Mills B. Building a personalized medicine infrastructure at a major cancer center. J Clin Oncol. 2013; 31: 1849-57.

168. Cerrone M, Napolitano C, Priori SG. Genetics of ion-channel disorders. Curr Opin Cardiol. 2012; 27: 242-52.

169. Moore JR, Leinwand L, Warshaw DM. Understanding cardiomyopathy phenotypes based on the functional impact of mutations in the myosin motor. Circ Res. 2012; 111: 375-85.

170. Fouchier SW, Dallinga-Thie GM, Meijers JC, Zelcer N, Kastelein JJ, Defesche JC, et al. Mutations in STAP1 are associated with autosomal dominant hypercholesterolemia. Circ Res. 2014; 115: 552-5.

171. Simino J, Kume R, Kraja AT, Turner ST, Hanis CL, Sheu WH, et al. Linkage analysis incorporating gene-age interactions identifies seven novel lipid loci: the Family Blood Pressure Program. Atherosclerosis. 2014; 235: 84-93.

172. Rosenthal EA, Ranchalis J, Crosslin DR, Burt A, Brunzell JD, Motulsky AG, et al. Joint linkage and association analysis with exome sequence data implicates SLC25A40 in hypertriglyceridemia Am J Hum Genet. 2013; 93: 1035-45.

173. Malandrino N, Smith RJ. Peronalized medicine in diabetes. Clin Chem. 2011; 57: 231-40

174. Smith RJ, Nathan DM, Arslanian SA, Groop L, Rizza RA, Rotter JI. Individualizing therapies in type 2 diabetes mellitus based on patient characteristics: what we know and what we need to know. J Clin Endocrinol Metab. 2010; 95: 1566-74. 
175. Muller G. Personalized prognosis and diagnosis of type 2 diabetes: vision or fiction? Pharmacology. 2010; 85: 168-87.

176. Wolkenhauer O, Fell D, De Meyts P, Bluthgen N, Herzel H, Le Novere N, et al. SysBioMed report: advancing systems biology for medical applications. IET Syst Biol. 2009; 3: 131-6.

177. Hood L, Heath JR, Phelps ME, Lin B. Systems biology and new technologies enable predictive and preventative medicine. Science. 2004; 306: 640-3 